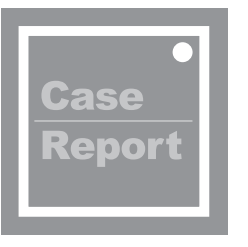

\title{
Stent-Assisted Coil Embolization for Acute Ruptured Dissecting Aneurysm at the P2 Segment of the Posterior Cerebral Artery: A Case Report
}

\author{
Takashi Mitsuhashi, ${ }^{1}$ Hidenori Oishi, ${ }^{2,3}$ Kensaku Makino, ${ }^{1}$ Joji Tokugawa, ${ }^{1}$ Atsuhito Fuse, ${ }^{4}$ and Makoto Hishii
}

Objective: We report a case of ruptured posterior cerebral artery (PCA) dissecting aneurysm treated with stent-assisted coil embolization in the acute phase of ruptured aneurysm.

Case Presentation: A60-year-old woman presented with sudden onset of severe headache followed by unconsciousness. CT showed severe subarachnoid hemorrhage. Digital subtraction angiography showed a dissecting aneurysm at the P2 segment of the right PCA. Stent-assisted coil embolization was performed for the ruptured dissecting aneurysm. Since thrombus was observed in the stent, ozagrel was administered intravenously, and the thrombus gradually disappeared during the follow-up period. She was discharged without neurological deficit.

Conclusion: Parent artery occlusion is widely performed for acute ruptured PCA dissecting aneurysm, but reconstruction with stent-assisted coiling is considered to be an effective therapeutic strategy.

Keywords $>$ posterior cerebral artery, dissecting aneurysm, stent-assisted coil embolization, subarachnoid hemorrhage

\section{Introduction}

Aneurysms arising from the posterior cerebral artery (PCA) are relatively rare and the reported incidence is less than $2 \%$ of all intracranial aneurysms. ${ }^{1)}$ PCA aneurysms are more frequently giant, dissecting, fusiform, or pseudoaneurysms compared to aneurysms located in other areas

${ }^{1}$ Department of Neurosurgery, Juntendo University Nerima Hospital, Tokyo, Japan

${ }^{2}$ Department of Neurosurgery, Faculty of Medicine, Juntendo University, Tokyo, Japan

${ }^{3}$ Department of Neuroendovascular Therapy, Faculty of Medicine, Juntendo University, Tokyo, Japan

${ }^{4}$ Department of Neurology, Juntendo University Nerima Hospital, Tokyo, Japan

Received: March 31, 2021; Accepted: June 8, 2021

Corresponding author: Takashi Mitsuhashi. Department of Neurosurgery, Juntendo University Nerima Hospital, 3-1-10, Takanodai, Nerima-ku, Tokyo 177-8521, Japan

Email: ta-kashi@wa2.so-net.ne.jp

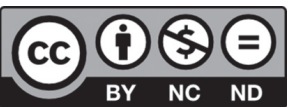

This work is licensed under a Creative Commons Attribution-NonCommercialNoDerivatives International License.

(C)2021 The Japanese Society for Neuroendovascular Therapy of the circle of Willis. ${ }^{2)}$ Saccular aneurysms of the PCA usually occur only at the P1 or the P1-P2 segment, whereas dissecting aneurysms of the PCA usually occur at the P2 or P2-P3 segment. ${ }^{3)}$ Dissecting aneurysms of the PCA can be posttraumatic or spontaneous. ${ }^{4)}$

Here, we report a case of ruptured PCA dissecting aneurysm to form two aneurysmal sacs, successfully treated with stent-assisted coil embolization in the acute phase. We also discuss the current status of stent-assisted coil embolization for this rare pathology.

\section{Case Presentation}

A previously healthy 60 -year-old woman presented to the emergency room with a sudden onset of severe headache followed by unconsciousness. She had no history of head trauma or connective tissue disease. Ocular examination found miosis but preserved light reflexes. Head CT demonstrated massive subarachnoid hemorrhage ( $\mathrm{SAH}$ ) mainly in the posterior fossa with acute hydrocephalus (Fig. 1A). The SAH was evaluated as Hunt and Hess grade V. After lumbar drainage, digital subtraction angiography revealed two consecutive irregular aneurysmal sacs at the P2 segment of the right PCA (Fig. 1B and 1C), which we 

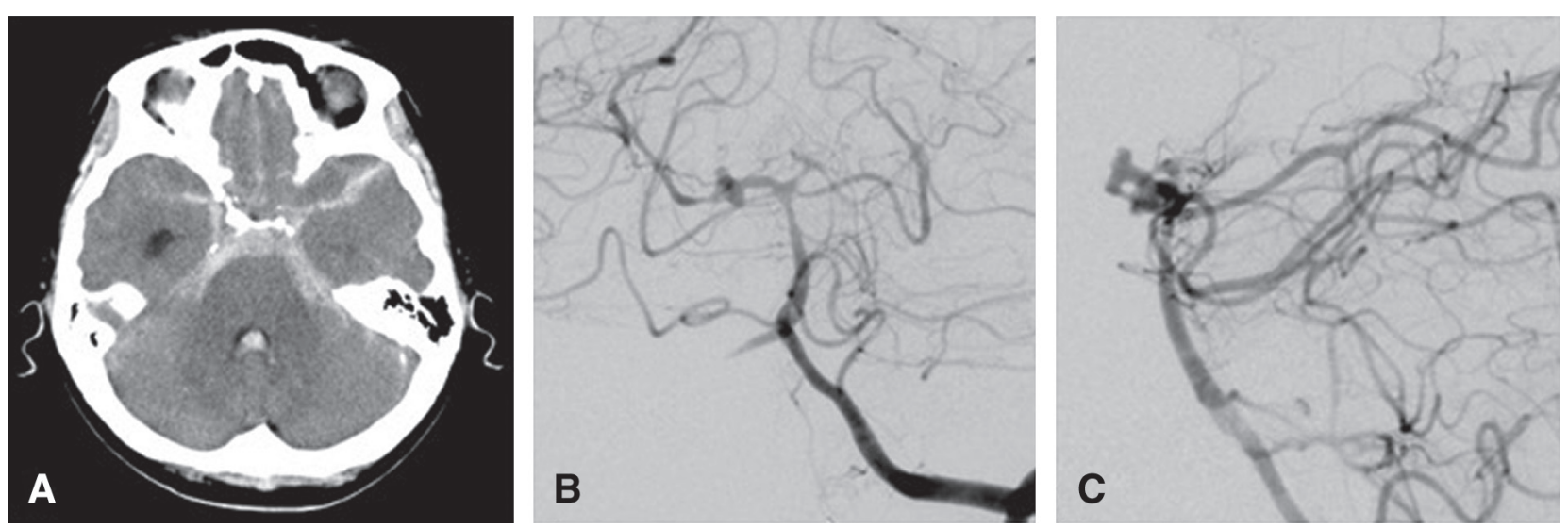

Fig. 1 Axial noncontrast cranial CT scan (A) showing diffuse thick SAH with intraventricular hemorrhage. Left vertebral angiograms, anteroposterior view (B), and lateral view (C) showing a

diagnosed as ruptured dissecting aneurysm. There was no significant difference in the shape of the two aneurysmal sacs, and it was not clear which aneurysm had ruptured.

We developed a treatment plan for stent-assisted coil embolization of each aneurysmal sac using the semi-jail technique, in which a microcatheter for stent deployment is placed distal to the dissecting aneurysm, another microcatheter is placed in the aneurysmal sac using the sheep technique, and the stent is deployed from the distal part of the dissecting aneurysm.

Endovascular surgery was performed under general anesthesia. A 6-Fr guiding catheter was placed in the cervical portion of the left vertebral artery, and then $4000 \mathrm{IU}$ of heparin was administered intravenously. $3 \mathrm{D}$ rotational angiography demonstrated two $2.5 \mathrm{~mm}$ aneurysms arising from both sides of the P2 segment of the right PCA (Fig. 2A and 2B). The proximal caliber of PCA was $1.81 \mathrm{~mm}$, and the distal caliber of PCA was $1.87 \mathrm{~mm}$. A Prowler Select Plus microcatheter (Codman Neurovascular, Raynham, MA, USA) was navigated into the distal portion of the right $\mathrm{P} 2$ (Fig. 2C and 2D), and a Headway Duo microcatheter (MicroVention, Aliso Viejo, CA, USA) was inserted into the dissecting aneurysm sac. Immediately after the procedure, aspirin $300 \mathrm{mg}$ and clopidogrel $300 \mathrm{mg}$ were administered via the nasogastric tube. We interrupted the procedure for about 10 minutes afterward to allow the antiplatelet agents to take effect. An Enterprise stent $4.0 \mathrm{~mm} / 16 \mathrm{~mm}$ (Johnson \& Johnson, Miami, FL, USA) was partially deployed from the distal end of the dissection to the proximal intact P1 segment of the PCA (Fig. 2E-2G). Two coils were inserted with stent protection and gradually unsheathed. The microcatheter was then inserted into the other aneurysm sac and two coils were delivered. After the dissecting aneurysm at the $\mathrm{P} 2$ segment of the right PCA. PCA: posterior cerebral artery; SAH: subarachnoid hemorrhage

delivery of the finishing coil in the proximal portion of the aneurysm sac, the stent was fully deployed. Both aneurysms were treated using the semi-jail technique. Since thrombus was observed in the stent (Fig. 2H), ozagrel sodium was administered intravenously, and the thrombus gradually disappeared during the follow-up period (Fig. 3).

Postprocedural dual-antiplatelet treatment of aspirin $100 \mathrm{mg}$ /day and clopidogrel $75 \mathrm{mg}$ /day was continued. The postoperative course was uneventful, and the patient was discharged without neurological deficit.

Six months have passed since the surgical procedure and the patient has no neurological deficit. Follow-up MRA showed no stent occlusion and good peripheral PCA delineation.

The patient showed no neurological deficit even after six months from the surgery, and follow-up MRA showed no stent occlusion and clear delineation of the distal PCA.

The use of an intracranial stent for acute ruptured cerebral aneurysm is not covered by Japan National Health Insurance, so was used after review by the Juntendo University Nerima Hospital ethics committee.

\section{Discussion}

PCA aneurysms are often associated with multiple aneurysms, arteriovenous malformations, complex angioarchitecture, and fusiform type. ${ }^{2)}$ In contrast, PCA dissecting aneurysm is extremely rare, occurs at a younger age, and shows female predominance, with only a few reported cases. $^{3-7)}$ The P2 segment is the most common location for PCA dissecting aneurysms. ${ }^{4,6)}$ Trauma or stress of the arterial wall of the PCA along the tentorial edge may be a cause of the development of PCA dissecting aneurysm. ${ }^{2,4}$ ) 

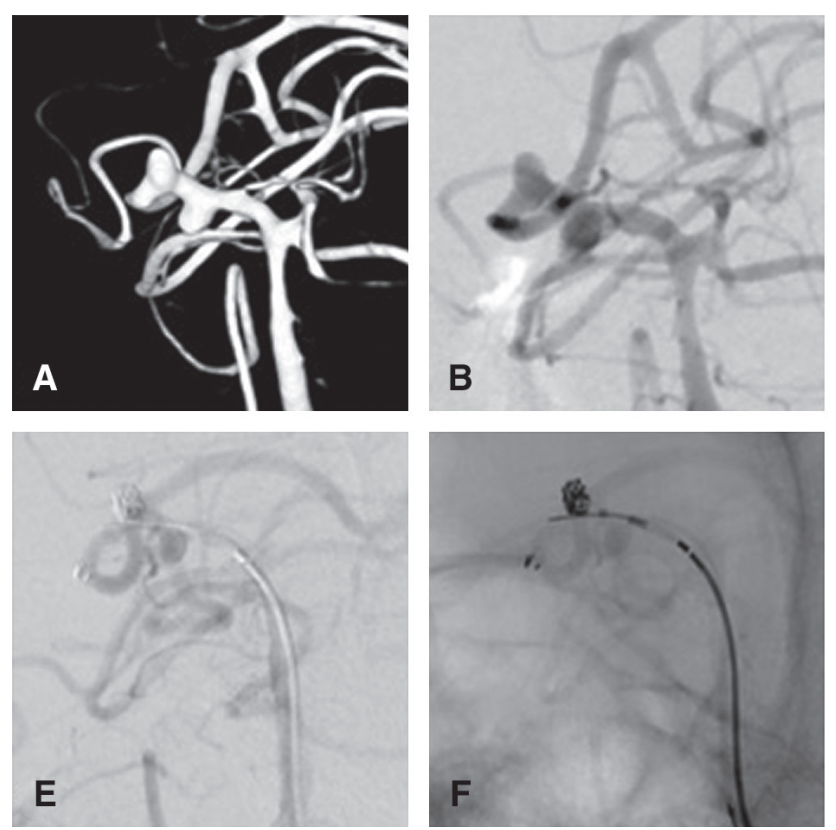

Fig. 23 D rotational angiogram of the working angle $(\mathbf{A})$ showing two 2.5-mm aneurysms arising from both sides of the P2 segment of the right PCA. Left vertebral angiogram of the working angle (B) showing the dissecting aneurysm at the $\mathrm{P} 2$ segment of the right PCA. First, the Prowler Select Plus microcatheter was navigated into the distal portion of the right P2 (C and D). After the microcatheter was
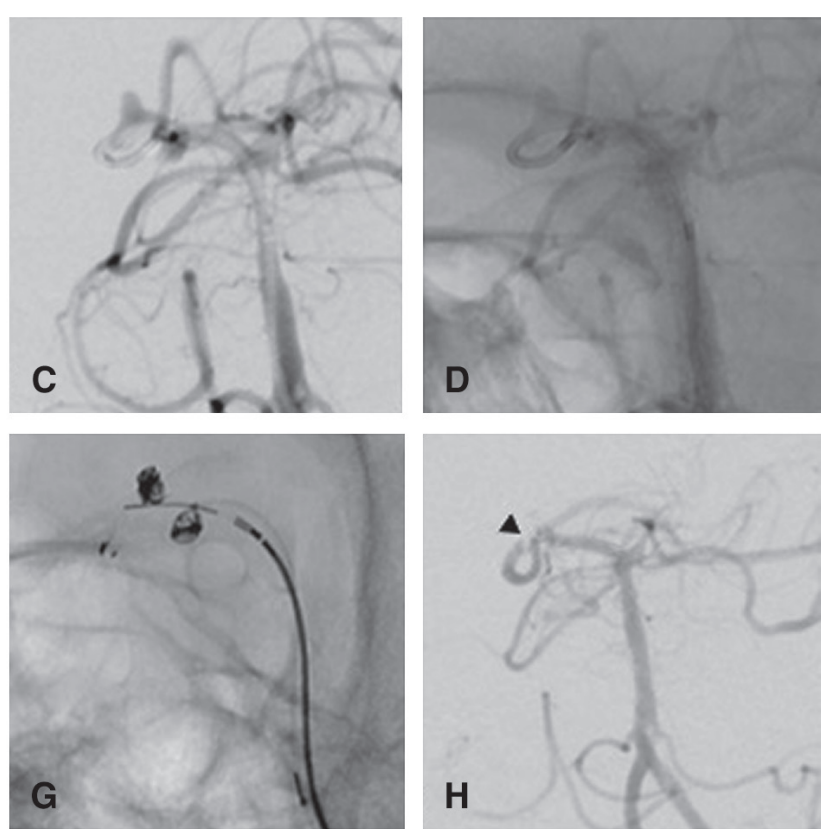

inserted into the dissecting aneurysm sac, an Enterprise stent was partially deployed from the distal end of the dissection to the proximal PCA. In addition, a microcatheter was placed in the other aneurysm sac, and then the Enterprise stent was fully deployed (E-G). Thrombus in the stent is recognized $(\mathbf{H}$; arrowhead). PCA: posterior cerebral artery

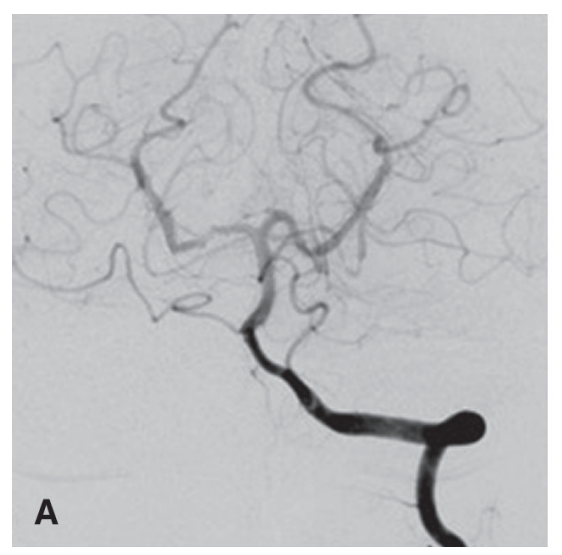

Fig. 3 Angiogram at 1 month after procedure, anteroposterior view (A), lateral view (B), and working angle (C) showing good obliteration

The PCA supplies parts of the temporal cortex, the calcarine, and occipital cortices, as well as parts of the brain stem and thalamus. The perforators of the PCA are divided into two types: the central perforating branches, including the thalamoperforating, thalamogeniculate, and peduncular perforating arteries, and the ventricular branches, including the posteromedial and posterolateral choroid arteries. ${ }^{7)}$ The central perforating branches usually originate from the P1 and/or P2 segments, and supply some important structures such as the diencephalon and midbrain. The P3 and P4 segments extend distally from the dorsal aspect of the midbrain and supply the calcarine and parieto-occipital cortices. Consequently, ischemia in the PCA territory including these various perfusion areas may result in a range of neurological disturbances such as memory disturbance, oculomotor palsy, visual disturbance (homonymous hemianopsia), and sometimes hemiparesis.

The treatment strategy of ruptured PCA dissecting aneurysms can involve either deconstruction or reconstruction. Deconstruction is based on parent artery occlusion (PAO), whereas reconstruction depends on stent-assisted coil embolization. Selective occlusion with preservation of the 
Mitsuhashi T, et al.

Table 1 Summary of cases of stent-assisted coil embolization for acute ruptured dissecting aneurysm at the P2 or P2-P3 segment of the PCA

\begin{tabular}{lccccccc}
\multicolumn{1}{c}{ Author (year) } & $\begin{array}{c}\text { Age } \\
\text { (years) }\end{array}$ & Sex & H\&H & Location & Event & Outcome & Stent \\
$\begin{array}{l}\text { Taqi et al. } \\
(2011)^{4)}\end{array}$ & 48 & F & Not mentioned & P2 & None & Not mentioned & Neuroform \\
& 54 & F & Not mentioned & P2-P3 & None & Not mentioned & Neuroform \\
& 23 & F & Not mentioned & P2 & Thrombus & Not mentioned & Neuroform \\
Wael Osman & 49 & F & 3 & P2 & None & 0 & Enterprise \\
$\begin{array}{l}\text { et al. (2017) } \\
\text { Choi et al. }\end{array}$ & 50 & F & 4 & P2-P3 & None & 5 & Enterprise \\
$\begin{array}{l}(2019)^{3)} \\
\text { Present case }\end{array}$ & 60 & F & 5 & P2 & Thrombus & 0 & Enterprise \\
\hline
\end{tabular}

F: female; H\&H: Hunt and Hess

parent artery is difficult for fusiform or giant serpentine aneurysms. ${ }^{8}$ Occlusion of the distal P2 segment can be tolerated under normal conditions due to the presence of collateral circulation. ${ }^{5)}$ Endovascular PCA sacrifice is also a safe method for managing P2 and distal aneurysms. ${ }^{9}$

The possibility that PAO could cause PCA territory infarction cannot be excluded. Therefore, PAO may lead to significant disability such as hemianopia or hemiplegia. One patient suffered from severe morbidity caused by occlusion of the $\mathrm{P} 1$ or proximal $\mathrm{P} 2$ in the presence of a rare variant of the posterior cerebral circulation characterized by a solitary arterial trunk that supplies both the paramedian thalami and the rostral midbrain. ${ }^{10)}$

Stent-assisted coiling has been widely used for the treatment of complex intracranial aneurysms, and numerous studies have demonstrated its favorable long-term efficacy. ${ }^{11)}$ In addition, stent-assisted coiling of complex PCA aneurysms resulted in favorable outcomes, with good complete occlusion rates and results comparable to aneurysms in other areas of the circle of Willis. ${ }^{12)}$ Therefore, stent-assisted coil embolization has recently been used for the treatment of acute ruptured PCA dissecting aneurysm. $3,4,6)$

The problem with stent-assisted coiling is the essential administration of antiplatelet agents prior to stenting even in the patients with acute ruptured aneurysms, which may increase the risk of hemorrhage, rupture, or extension of the lesion. Furthermore, long-term antiplatelet therapy is essential, especially in patients with acute ruptured aneurysms who are at risk for hemorrhagic events. Such patients may also require surgical procedures such as external ventricular drainage and ventriculoperitoneal shunting. Stent-assisted coiling for acute ruptured aneurysms has a 10-fold higher complication rate than for unruptured aneurysms, ${ }^{13)}$ and a $13 \%$ higher rate of severe disability and mortality due to the higher complication rate. ${ }^{14)}$
The timing of antiplatelet administration during stentassisted coil embolization is important, as preoperative loading reduces the incidence of perioperative ischemic complications, whereas preoperative loading in the acute phase of ruptured aneurysm carries increased risk of bleeding complications. ${ }^{15)}$ In the present case, aspirin and clopidogrel loading was performed immediately before intraoperative stent deployment, but in-stent thrombosis occurred. The effect of the antiplatelet therapy may have been inadequate, but we believe that the administration immediately before stent deployment in patients with acute ruptured aneurysm will avoid worsening of bleeding complications.

In 2020, the Delphi consensus statement described on antiplatelet management for stent-assisted coiling and flow diversion of ruptured intracranial aneurysms. ${ }^{16)}$ In the statement, the first-line antiplatelet therapy is intravenous aspirin $500 \mathrm{mg}$ bolus injection or oral aspirin $75-325 \mathrm{mg}$. The second choice of antiplatelet agent is the glycoprotein $\mathrm{IIb} / \mathrm{III}$ a receptor inhibitor, and if this is not available, the oral administration of $180 \mathrm{mg}$ of ticagrelor. Furthermore, the timing of intracranial device (stents or flow diverters) placement is considered to be immediately after the administration of antiplatelet agent. Since glycoprotein IIb/IIIa receptor inhibitor and ticagrelor are not available in Japan or not covered by Japan National Health Insurance, we substituted aspirin and clopidogrel loading.

Five cases of stent-assisted coil embolization for acute ruptured dissecting aneurysm at the P2 or P2-P3 segment of the PCA have been reported (Table 1)., ${ }^{3,46}$ All patients were female, with a mean age of 44.8 years. Our patient was also female, but she was aged 60 years, considerably older than previous patients. One patient with intraoperative thrombus developing at the stent was treated with intra-arterial abciximab, resulting in resolution of the 
thrombus. In our case, intra-stent thrombosis was observed intraoperatively and was resolved with intravenous administration of ozagrel sodium. Ozagrel inhibits platelet aggregation by markedly suppressing thromboxane $\mathrm{A}_{2}$ production and increasing prostacyclin production, and intravenous administration has rapid antiplatelet effects. ${ }^{17)}$

One patient had delayed growth of the aneurysm and was subsequently treated with PAO, but the other four patients did not require further treatment. The delayed growth of the aneurysm may have been caused by the difficulty in identifying the exact dissecting segment, as suggested previously. ${ }^{18)}$

The stents used for stent-assisted coil embolization were Neuroform (Boston Scientific, Marlborough, MA, USA) in three cases and Enterprise in two. Both stents are laser cut types with a highly flexible closed-cell design that is easy to deploy and exert a high radial force to stabilize the coil in the aneurysm. ${ }^{19)}$ Recently, a case of acute ruptured PCA dissecting aneurysm was treated with the Pipeline embolization device (Medtronic, Minneapolis, MN, USA). ${ }^{20)}$ However, the use of the flow diverter in the acute phase of a ruptured aneurysm remains controversial. In many cases in the acute rupture phase, antiplatelet agents are difficult to administer preoperatively, and the risk of intraoperative thrombus is considered to be high.

\section{Conclusion}

We successfully treated a ruptured PCA dissecting aneurysm in the acute phase with stent-assisted coil embolization. $\mathrm{PAO}$ is widely performed for acute ruptured PCA dissecting aneurysm, but reconstruction with stent-assisted coiling is considered to be an effective therapeutic strategy.

\section{Informed Consent}

Informed consent has been obtained from the patient for this report.

\section{Disclosure Statement}

The authors declare that there is no conflict of interest.

\section{References}

1) Goehre F, Jahromi BR, Lehecka M, et al. Posterior cerebral artery aneurysms: treatment and outcome analysis in 121 patients. World Neurosurg 2016; 92: 521-532.
2) Goehre F, Jahromi BR, Hernesniemi J, et al. Characteristics of posterior cerebral artery aneurysms: an angiographic analysis of 93 aneurysms in 81 patients. Neurosurgery 2014; 75: 134-144; discussion 143-144; quiz 144.

3) Choi S, Chung J. Endovascular treatment of ruptured dissecting aneurysms on the P2-3 segment of the posterior cerebral artery: a report of two cases. $J$ Neurointensive Care 2019; 2: 87-92.

4) Taqi MA, Lazzaro MA, Pandya DJ, et al. Dissecting aneurysms of posterior cerebral artery: clinical presentation, angiographic findings, treatment, and outcome. Front Neurol 2011; 2: 38.

5) Liu L, He H, Jiang C, et al. Deliberate parent artery occlusion for non-saccular posterior cerebral artery aneurysms. Interv Neuroradiol 2011; 17: 159-168.

6) Wael Osman M, Kadziolka K, Peirot L. Optional endovascular therapy of dissecting posterior cerebral artery aneurysm. Interv Neurol 2017; 6: 219-228.

7) Párraga RG, Ribas GC, Andrade SE, et al. Microsurgical anatomy of the posterior cerebral artery in threedimensional images. World Neurosurg 2011; 75: 233-257.

8) Qin X, Xu F, Maimaiti Y, et al. Endovascular treatment of posterior cerebral artery aneurysms: a single center's experience of 55 cases. J Neurosurg 2017; 126: 1094-1105.

9) $\mathrm{Xu} \mathrm{J}, \mathrm{Xu} \mathrm{L}, \mathrm{Wu} \mathrm{Z}$, et al. Fetal-type posterior cerebral artery: the pitfall of parent artery occlusion for ruptured P2 segment and distal aneurysms. J Neurosurg 2015; 123: 906-914.

10) Sturiale CL, De Waure C, Della Pepa GM, et al. Endovascular treatment of the posterior cerebral artery aneurysms: single-center experience and a systematic review. World Neurosurg 2016; 91: 154-162.

11) Feng MT, Wen WL, Feng ZZ, et al. Endovascular embolization of intracranial aneurysms: to use stent(s) or not? Systematic review and meta-analysis. World Neurosurg 2016; 93: 271-278.

12) Xue G, Tang H, Liu P, et al. Safety and long-term efficacy of stent-assisted coiling for the treatment of complex posterior cerebral artery aneurysms. Clin Neuroradiol 2021; 31 : 89-97.

13) Bechan RS, Sprengers ME, Majoie CB, et al. Stent-assisted coil embolization of intracranial aneurysms: complications in acutely ruptured versus unruptured aneurysms. AJNR Am J Neuroradiol 2016; 37: 502-507.

14) Chalouhi N, Jabbour P, Singhal S, et al. Stent-assisted coiling of intracranial aneurysms: predictors of complications, recanalization, and outcome in 508 cases. Stroke 2013; 44: 1348-1353.

15) Golshani K, Ferrel A, Lessne M, et al. Stent-assisted coil emboilization of ruptured intracranial aneurysms: a retrospective multicenter review. Surg Neurol Int 2012; 3: 84.

16) Ospel JM, Brouwer P, Dorn F, et al. Antiplatelet management for stent-assisted coiling and flow diversion of 
ruptured intracranial aneurysms: a Delphi consensus statement. AJNR Am J Neuroradiol 2020; 41: 1856-1862.

17) Zhang J, Yang J, Chang $X$, et al. Ozagrel for acute ischemic stroke: a meta-analysis of data from randomized controlled trials. Neurol Res 2012; 34: 346-353.

18) Park W, Kwon DH, Ahn JS, et al. Treatment strategies for dissecting aneurysms of the posterior cerebral artery. Acta Neurochir (Wien) 2015; 157: 1633-1643.
19) Kadkhodayan Y, Rhodes N, Blackburn S, et al. Comparison of Enterprise with neuroform stent-assisted coiling of intracranial aneurysms. AJR Am J Roentgenol 2013; 200: 872-878.

20) Cunegatto-Braga M, Hogan B, Aguilar-Salinas $P$, et al. Pipeline embolization device flow diversion for a dissecting ruptured posterior cerebral artery aneurysm in a pediatric patient. World Neurosurg 2018; 117: 255-260. 